\title{
A Rainbow $k$-Matching in the Complete Graph with $r$ Colors
}

\author{
Shinya Fujita ${ }^{1 *}$ Atsushi Kaneko, Ingo Schiermeyer ${ }^{2}$, \\ and Kazuhiro Suzuki ${ }^{3 \dagger}$
}

1 Department of Mathematics, Gunma National College of Technology, 580 Toriba, Maebashi, Gunma, 371-8530 Japan, shinyaa@mti.biglobe.ne.jp

${ }^{2}$ Institut für Diskrete Mathematik und Algebra,

Technische Universität Bergakademie Freiberg, 09596 Freiberg, Germany, schierme@math.tu-freiberg.de

${ }^{3}$ Department of Computer and Information Sciences, Ibaraki University, Hitachi, Ibaraki, 316-8511 Japan, tutetuti@dream.com

Submitted: Dec 31, 2007; Accepted: Apr 18, 2009; Published: Apr 30, 2009

Mathematics Subject Classifications: $05 \mathrm{C} 15 \ddagger 05 \mathrm{C} 70^{\S}$

\begin{abstract}
An $r$-edge-coloring of a graph is an assignment of $r$ colors to the edges of the graph. An exactly $r$-edge-coloring of a graph is an $r$-edge-coloring of the graph that uses all $r$ colors. A matching of an edge-colored graph is called rainbow matching, if no two edges have the same color in the matching. In this paper, we prove that an exactly $r$-edge-colored complete graph of order $n$ has a rainbow matching of size $k(\geq 2)$ if $r \geq \max \left\{\left(\begin{array}{c}2 k-3 \\ 2\end{array}\right)+2,\left(\begin{array}{c}k-2 \\ 2\end{array}\right)+(k-2)(n-k+2)+2\right\}, k \geq 2$, and $n \geq 2 k+1$. The bound on $r$ is best possible.
\end{abstract}

Keyword(s): edge-coloring, matching, complete graph, anti-Ramsey, rainbow, heterochromatic, totally multicolored

\section{Introduction}

We consider finite, undirected, simple graphs $G$ with the vertex set $V(G)$ and the edge set $E(G)$. An r-edge-coloring of a graph $G$ is a mapping color $: E(G) \rightarrow C$, where $C$ is

\footnotetext{
${ }^{*}$ Corresponding author

${ }^{\dagger}$ Present affiliation is Department of Electronics and Informatics Frontier, Kanagawa University, Yokohama, Kanagawa, 221-8686 Japan.

${ }^{\ddagger} 05 \mathrm{C} 15$ Coloring of graphs and hypergraphs

${ }^{\S} 05 \mathrm{C} 70$ Factorization, matching, covering and packing
} 
a set of $r$ colors. An exactly $r$-edge-coloring of a graph is an $r$-edge-coloring of the graph such that all $r$ colors is used, namely, every color appears in the $r$-edge-colored graph. A subgraph $H$ of an edge-colored graph is said to be rainbow (or heterochromatic, or totally multicolored) if no two edges of $H$ have the same color, that is, if $\operatorname{color}(e) \neq \operatorname{color}(f)$ for any two distinct edges $e$ and $f$ of $H$. A matching of size $k$ is called a $k$-matching. Let $P_{k}$ and $C_{k}$ are the path and the cycle of order $k$, respectively.

We begin with a brief introduction of the background concerning anti-Ramsey numbers. Let $h_{p}(n)$ be the minimum number of colors $r$ such that every exactly $r$-edge-colored complete graph $K_{n}$ contains a rainbow $K_{p}$. The pioneering paper [2] by Erdös, Simonovits and Sós proved the existence of a number $n_{0}(p)$ such that $h_{p}(n)=t_{p-1}(n)+2$ for $n>n_{0}(p)$, where $t_{p-1}(n)$ is the Turán number. Montellano-Ballesteros and Neumann-Lara [5] proved that for all integers $n$ and $p$ such that $3 \leq p<n$, the corresponding anti-Ramsey function is such. Along a slightly different line, Eroh [3,4] studied rainbow Ramsey numbers for matchings, which is a certain generalization of the Ramsey and anti-Ramsey numbers. For two graphs $G_{1}$ and $G_{2}$, let $R M\left(G_{1}, G_{2}\right)$ be the minimum integer $n$ such that any edge-coloring of $K_{n}$ contains either a monochromatic $G_{1}$ or a rainbow $G_{2}$. In [3], the case where $G_{1}$ is a star and $G_{2}$ is a matching is discussed. Also, in [4], the case where each $G_{i}$ with $i=1,2$ is a $k_{i}$-matching is treated. There, in particular, it is conjectured that $R M\left(G_{1}, G_{2}\right)=k_{2}\left(k_{1}-1\right)+2$, and a proof in the case where $k_{2} \leq \frac{3}{2}\left(k_{1}-1\right)$ is given.

In this paper, we study anti-Ramsey numbers for $k$-matchings. Given an exactly $r$ edge-colored complete graph of order $n$, is there a rainbow $k$-matching? Since the case $k=0$ and $k=1$ is trivial, we assume $k \geq 2$. For example, if $n=7$ and $r \geq 2$ then we can find easily a rainbow 2-matching, but we may not find a rainbow 3-matching. Generally, if $n \geq 2 k+1$ then the following colorings do not allow a rainbow $k$-matching to exist. (See Figure 1.)

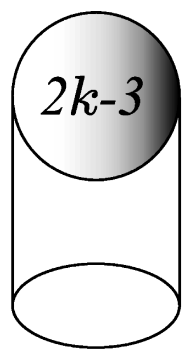

(a)

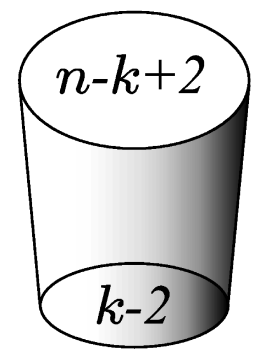

(b)

Figure 1: Colorings without rainbow $k$-matchings.

In the coloring $(a)$ of Figure 1 , a complete subgraph $K_{2 k-3}$ of $G$ is rainbow and the other edges are colored with exactly one color, namely, monochromatic. In the coloring (b), a complete subgraph $K_{n-(k-2)}$ of $G$ is monochromatic and the other edges are rainbow. In each coloring, it is clear that there is no rainbow $k$-matching. However, if there are more colors than in these colorings, is there a rainbow $k$-matching ? Schiermeyer [6] solved this problem affirmatively for $k \geq 2$ and $n \geq 3 k+3$. In this paper, we solve this 
problem for $n \geq 2 k+1$.

Theorem 1.1. An exactly $r$-edge-colored complete graph of order $n$ has a rainbow $k$ matching, if $r \geq \max \left\{\left(\begin{array}{c}2 k-3 \\ 2\end{array}\right)+2,\left(\begin{array}{c}k-2 \\ 2\end{array}\right)+(k-2)(n-k+2)+2\right\}, k \geq 2$, and $n \geq 2 k+1$.

If $n=2 k$ then there exists an exactly $r$-edge-coloring with $r=\left(\begin{array}{c}2 k-3 \\ 2\end{array}\right)+2$ for $k \geq 3$ or $r=\left(\begin{array}{c}2 k-3 \\ 2\end{array}\right)+3$ for $k=2$ such that there is no rainbow $k$-matching. (See Figure 2.)

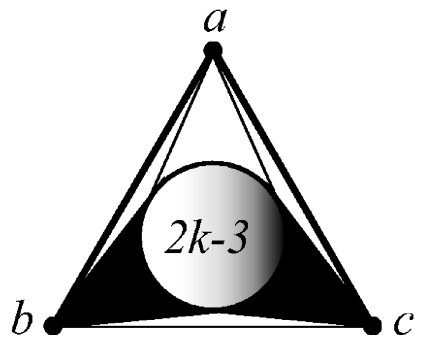

(a)

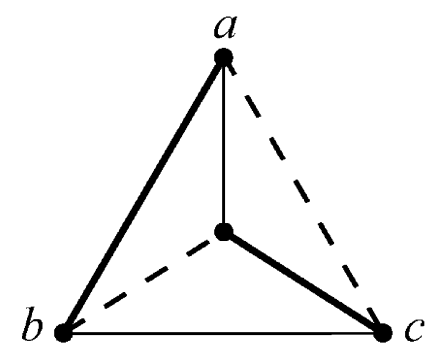

(b)

Figure 2: $\left(\left(\begin{array}{c}2 k-3 \\ 2\end{array}\right)+2\right.$ or +3$)$-Colorings without rainbow $k$-matchings.

In the coloring $(a)$ of Figure 2, a complete subgraph $K_{2 k-3}$ of $G$ is rainbow and the other edges are colored with exactly two colors red and blue, so that $a b, a c$ and the edges between $\{b, c\}$ and $G-\{a, b, c\}$ are red, and $b c$ and the edges between $a$ and $G-\{a, b, c\}$ are blue. Thus, the number of colors is $\left(\begin{array}{c}2 k-3 \\ 2\end{array}\right)+2$, but there is no rainbow 1-factor. In the coloring $(b)$ of Figure 2, $K_{4}$ is colored with three colors. Then, $k=2,\left(\begin{array}{c}2 k-3 \\ 2\end{array}\right)+3=3$, and $\left(\begin{array}{c}k-2 \\ 2\end{array}\right)+(k-2)(n-k+2)+2=2$, but any 1 -factor is monochromatic. We propose the following conjecture.

Conjecture 1.2. An exactly $r$-edge-colored complete graph of order $2 k(\geq 6)$ has a rainbow 1-factor, if $r \geq \max \left\{\left(\begin{array}{c}2 k-3 \\ 2\end{array}\right)+3,\left(\begin{array}{c}k-2 \\ 2\end{array}\right)+k^{2}-2\right\}$.

We have proved that this conjecture holds for $3 \leq k \leq 4$ in our preprint (we can send the proof upon request), but for $k \geq 5$ this is still open.

The concept of rainbow matchings is linked with the relationship between the maximum number of edges and the edge independence number in graphs. In 1959, Erdös and Gallai [1] proved the following theorem.

Theorem 1.3 ([1]). Let $G$ be a graph of order $n \geq 2 k+1$ with edge independence number at most $k$. Then $|E(G)| \leq \max \left\{\left(\begin{array}{c}2 k+1 \\ 2\end{array}\right),\left(\begin{array}{l}k \\ 2\end{array}\right)+k(n-k)\right\}$.

In fact, Theorem 1.1 nearly implies Theorem 1.3, that is, the following corollary is obtained by Theorem 1.1.

Corollary 1.4. If $n \geq 2 k+5$, then the assertion of Theorem 1.3 follows from Theorem 1.1. 
Proof. Color the edges of the complete graph $K_{n}$ of order $n \geq 2 k+5=2(k+2)+1$, so that, a spanning subgraph $H$ isomorphic to $G$ is rainbow and the other edges are colored with one new color. Then the number of colors $r$ is $|E(H)|+1=|E(G)|+1$. Since the edge independence number of $H$ is at most $k, H$ has no rainbow $(k+1)$-matching. Thus, $K_{n}$ has no rainbow $(k+2)$-matching. Hence, by Theorem 1.1, $r \leq \max \left\{\left(\begin{array}{c}2(k+2)-3 \\ 2\end{array}\right)+\right.$ $\left.1,\left(\begin{array}{c}(k+2)-2 \\ 2\end{array}\right)+((k+2)-2)(n-(k+2)+2)+1\right\}=\max \left\{\left(\begin{array}{c}2 k+1 \\ 2\end{array}\right)+1,\left(\begin{array}{l}k \\ 2\end{array}\right)+k(n-k)+1\right\}$. Therefore, $|E(G)|=r-1 \leq \max \left\{\left(\begin{array}{c}2 k+1 \\ 2\end{array}\right),\left(\begin{array}{c}k \\ 2\end{array}\right)+k(n-k)\right\}$.

In the next section, we give the proof of Theorem 1.1. In the rest of this section, we introduce some notation for the proof of the theorem. For a graph $G$ and a vertex subset $M$ of $V(G)$, let $G[M]$ denote the induced subgraph by $M$. For an element $x$ of a set $S$, we denote $S-\{x\}$ by $S-x$. For a matching $M$ and edges $e_{1}, \ldots, e_{k}, f_{1}, \ldots, f_{l}$, we denote $\left(M-\left\{e_{1}, \ldots, e_{k}\right\}\right) \cup\left\{f_{1}, \ldots, f_{l}\right\}$ by $M-e_{1}-\cdots-e_{k}+f_{1}+\cdots+f_{l}$. We often denote an edge $e=\{x, y\}$ by $x y$ or $y x$. For an edge-colored graph $G$ and an edge set $E \subseteq E(G)$, we define $\operatorname{color}(E)=\{\operatorname{color}(e) \mid e \in E\}$.

\section{Proof of Theorem 1.1}

Proof. Let $G$ be an exactly $r$-edge-colored complete graph of order $n \geq 2 k+1$ with no rainbow $k$-matchings. We may assume that $r$ is chosen as large as possible under the above assumption. To prove the theorem, it suffices to show that

$$
r<\max \left\{\left(\begin{array}{c}
2 k-3 \\
2
\end{array}\right)+2,\left(\begin{array}{c}
k-2 \\
2
\end{array}\right)+(k-2)(n-k+2)+2\right\} .
$$

We begin with the following basic Claim.

Claim 1. G has a rainbow $(k-1)$-matching.

Proof. We may assume that $G$ is not rainbow, because the complete graph of order at least $2 k$ has a $k$-matching. Hence, there are two edges $e, f$ such that $\operatorname{color}(e)=\operatorname{color}(f)$. Change the color of $e$ into the $(r+1)$-th new color. Then, by the maximality of $r$, there is a rainbow $k$-matching $M_{k}$. Therefore, $M_{k}-e$ is a desired rainbow matching of $G$, because $\left|M_{k}-e\right| \geq k-1$.

Let $M=\left\{e_{1}, e_{2}, \ldots, e_{k-1}\right\}$ be a rainbow $(k-1)$-matching of $G$. Let $x_{i}$ and $y_{i}$ be the end vertices of $e_{i}$, namely $e_{i}=x_{i} y_{i}$. Remove these vertices $x_{i}$ and $y_{i}$, and let $H$ be the resulting graph, namely $H=G-\bigcup_{1 \leq i \leq k-1}\left\{x_{i}, y_{i}\right\}$. Since $n \geq 2 k+1$, we have $|V(H)| \geq 3$. Hence $E(H) \neq \emptyset$.

Claim 2. $\operatorname{color}(E(H)) \subseteq \operatorname{color}(M)$.

Proof. If $\operatorname{color}(E(H)) \nsubseteq \operatorname{color}(M)$, then we have a rainbow $k$-matching $M+e$ of $G$ where $e$ is an edge of $H$ with color $(e) \in \operatorname{color}(E(H))-\operatorname{color}(M)$, which is a contradiction. 
Without loss of generality, we may assume $\operatorname{color}(E(H))=\left\{\operatorname{color}\left(e_{1}\right), \operatorname{color}\left(e_{2}\right), \ldots\right.$, color $\left.\left(e_{p}\right)\right\}$ for some positive integer $p \leq k-1$. Since $E(H) \neq \emptyset$, note that $1 \leq p$. Let $M_{1}=\left\{e_{1}, e_{2}, \ldots, e_{p}\right\}$ and $M_{2}=M-M_{1}$. (See Figure 3.)

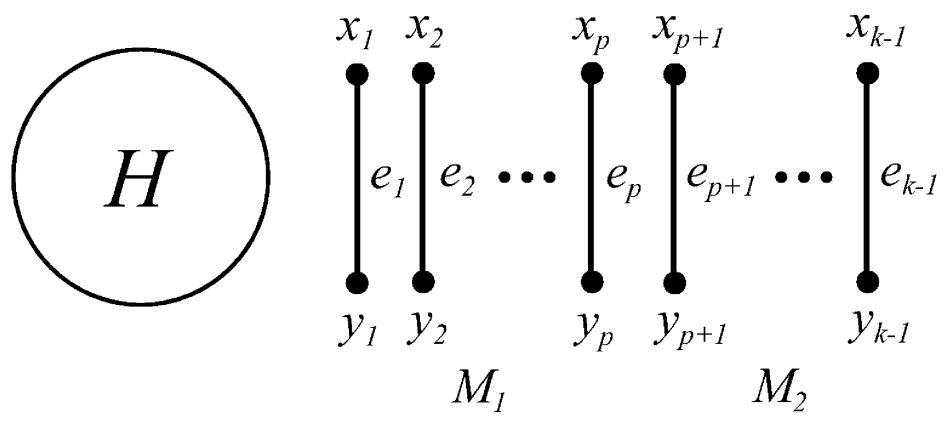

Figure 3: $H$ and $M=M_{1} \cup M_{2}$.

Let $G^{\prime}$ be a rainbow exactly $r$-edge-colored spanning subgraph of $G$ that contains $M$. Since $G^{\prime}$ is rainbow and $G^{\prime}$ contains $M$, note that $E\left(G^{\prime}\right) \cap E(H)=\emptyset$ (i.e., $H$ induces isolated vertices in $G^{\prime}$ ). Here, we would like to count the number of colors in $G$. It is enough to count the number of edges of $G^{\prime}$ because $|\operatorname{color}(E(G))|=\left|\operatorname{color}\left(E\left(G^{\prime}\right)\right)\right|=$ $\left|E\left(G^{\prime}\right)\right|$. Below, we consider only $G^{\prime}$ and the edges of $H$. Here, we give some notation. For two disjoint vertex sets $A$ and $B$, we define $E^{\prime}(A, B)=\left\{a b \in E\left(G^{\prime}\right) \mid a \in A, b \in B\right\}$. In the rest of the proof, for an edge $e=a b, a b$ is often regarded as its vertex set $\{a, b\}$ when there is no fear of confusion.

Claim 3. For any two distinct edges $e_{i} \in M_{1}$ and $e_{j} \in M,\left|E^{\prime}\left(e_{i}, e_{j}\right)\right| \leq 2$.

Proof. By the definition of $M_{1}$, there exists an edge $f_{1} \in E(H)$ such that $\operatorname{color}\left(f_{1}\right)=$ color $\left(e_{i}\right)$. If $\left|E^{\prime}\left(e_{i}, e_{j}\right)\right| \geq 3$ then there are two independent edges $f_{2}$ and $f_{3}$ in $E^{\prime}\left(e_{i}, e_{j}\right)$. Since $G^{\prime}$ contains $M$ and $G^{\prime}$ is rainbow, color $\left(f_{2}\right), \operatorname{color}\left(f_{3}\right) \notin \operatorname{color}(M)$ and $\operatorname{color}\left(f_{2}\right) \neq$ $\operatorname{color}\left(f_{3}\right)$. Since color $\left(f_{1}\right)=\operatorname{color}\left(e_{i}\right), \operatorname{color}\left(f_{1}\right) \neq \operatorname{color}\left(f_{2}\right)$ and color $\left(f_{1}\right) \neq \operatorname{color}\left(f_{3}\right)$. Hence, we have a rainbow $k$-matching $M-e_{i}-e_{j}+f_{1}+f_{2}+f_{3}$, which is a contradiction.

Claim 4. For any edge $e_{i} \in M_{1}$, let $g_{i}$ be an edge in $E(H)$ such that color $\left(e_{i}\right)=\operatorname{color}\left(g_{i}\right)$. Then $E^{\prime}\left(e_{i}, V(H)\right)=E^{\prime}\left(e_{i}, g_{i}\right)$ holds.

Proof. Suppose that for some edge $f_{1} \in E(H)$ with $\operatorname{color}\left(e_{i}\right)=\operatorname{color}\left(f_{1}\right)$ and for some edge $f_{2} \in E^{\prime}\left(e_{i}, V(H)\right)$, these edges $f_{1}, f_{2}$ are independent. (See Figure 4.) By the definition of $G^{\prime}, \operatorname{color}\left(f_{2}\right) \notin \operatorname{color}(M)$. Thus, we have a rainbow $k$-matching $M-e_{i}+$ $f_{1}+f_{2}$, which is a contradiction. From this observation, the claim follows.

Claim 5. If $E^{\prime}\left(e_{i}, V(H)\right) \neq \emptyset$ for an edge $e_{i} \in M_{1}$, then the color of $e_{i}$ induces a star in the graph $H$. 


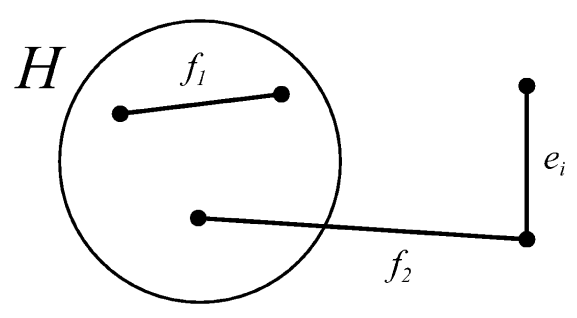

Figure 4: $f_{1}, f_{2}$ are independent.

Proof. Let $a b \in E^{\prime}\left(e_{i}, V(H)\right)$ such that $b \in V(H)$. By Claim 4, all the edges of $H$ which have $\operatorname{color}\left(e_{i}\right)$ in common are adjacent to the vertex $b$. Hence, the color of $e_{i}$ induces a star with the center $b$ in the graph $H$.

Claim 6. If $H$ has a rainbow 2-matching $f_{1}$ and $f_{2}$ then $E^{\prime}\left(e_{i}, e_{j}\right)=\emptyset$ for some edges $e_{i}, e_{j} \in M_{1}$ such that color $\left(f_{1}\right)=\operatorname{color}\left(e_{i}\right)$ and $\operatorname{color}\left(f_{2}\right)=\operatorname{color}\left(e_{j}\right)$.

Proof. By Claim 2, there are some edges $e_{i}, e_{j} \in M_{1}$ such that $\operatorname{color}\left(f_{1}\right)=\operatorname{color}\left(e_{i}\right)$ and $\operatorname{color}\left(f_{2}\right)=\operatorname{color}\left(e_{j}\right)$. Suppose that $E^{\prime}\left(e_{i}, e_{j}\right) \neq \emptyset$. Let $f_{3} \in E^{\prime}\left(e_{i}, e_{j}\right)$. Then we have a rainbow $k$-matching $M-e_{i}-e_{j}+f_{1}+f_{2}+f_{3}$, which is a contradiction.

Claim 7. For any edge $e_{i} \in M_{1},\left|E^{\prime}\left(e_{i}, V(H)\right)\right| \leq 2$.

Proof. By the definition of $M_{1}$, there exists an edge $f_{1} \in E(H)$ such that color $\left(f_{1}\right)=$ color $\left(e_{i}\right)$. By Claim $4, E^{\prime}\left(e_{i}, V(H)\right)=E^{\prime}\left(e_{i}, f_{1}\right)$. If $\left|E^{\prime}\left(e_{i}, V(H)\right)\right| \geq 3$, that is, $\left|E^{\prime}\left(e_{i}, f_{1}\right)\right|$ $\geq 3$, then there are two independent edges $f_{2}$ and $f_{3}$ in $E^{\prime}\left(e_{i}, f_{1}\right)$ By the definition of $G^{\prime}, \operatorname{color}\left(f_{2}\right), \operatorname{color}\left(f_{3}\right) \notin \operatorname{color}(M)$ and color $\left(f_{2}\right) \neq \operatorname{color}\left(f_{3}\right)$. Hence, we have a rainbow $k$-matching $M-e_{i}+f_{2}+f_{3}$, which is a contradiction.

Let $V_{1}=\bigcup_{x y \in M_{1}}\{x, y\}$ and $V_{2}=\bigcup_{x y \in M_{2}}\{x, y\}$. (See Figure 5.) We count the number of edges in $G^{\prime}-V_{2}$.

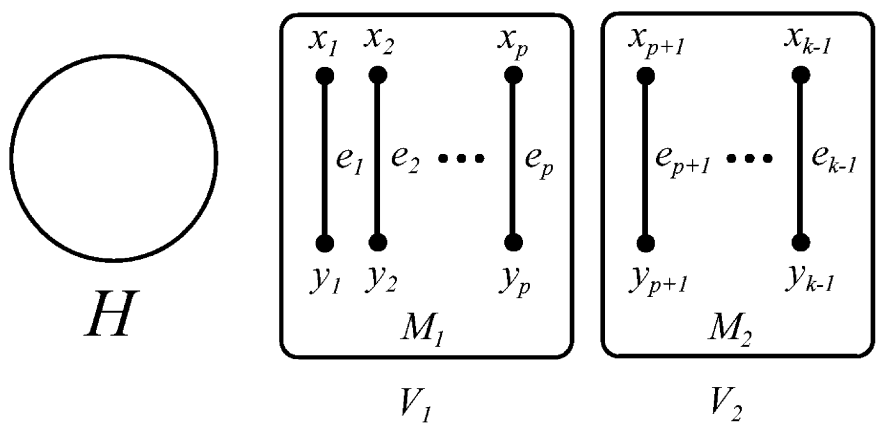

Figure 5: $H$ and $V_{1}, V_{2}$.

Claim 8. $\left|E\left(G^{\prime}-V_{2}\right)\right| \leq 2\left(\begin{array}{l}p \\ 2\end{array}\right)+3 p-2$. 
Proof. By the definition of $G^{\prime}, G^{\prime}$ has no edges of $H$. Hence, by Claim 3 and Claim 7, we have $\left|E\left(G^{\prime}-V_{2}\right)\right|=\left|E\left(G^{\prime}\left[V_{1}\right]\right)\right|+\left|E^{\prime}\left(V_{1}, V(H)\right)\right| \leq\left|M_{1}\right|+2\left(\begin{array}{c}p \\ 2\end{array}\right)+2 p=2\left(\begin{array}{c}p \\ 2\end{array}\right)+3 p$. Then, in view of the above inequality, it suffices to show that there exists some edge $e_{i} \in M_{1}$ such that $E^{\prime}\left(e_{i}, e_{j}\right)=\emptyset$ for some edge $e_{j} \in M_{1}$ with $j \neq i$ or $E^{\prime}\left(e_{i}, V(H)\right)=\emptyset$.

Suppose that for any edges $e_{i}, e_{j} \in M_{1}, E^{\prime}\left(e_{i}, e_{j}\right) \neq \emptyset$ and $E^{\prime}\left(e_{i}, V(H)\right) \neq \emptyset$. If $|V(H)| \geq 4$ then by Claim $5, H$ has a rainbow 2-matching. Thus, by Claim 6 , there exist some edges $e_{i}, e_{j} \in M_{1}$ such that $E^{\prime}\left(e_{i}, e_{j}\right)=\emptyset$, which is a contradiction. Therefore, we have $|V(H)|=3$. Then, $H$ is a triangle $\{a, b, c\}$. Hence it follows that $p=\left|M_{1}\right|=1,2$, or 3 because color $(E(H))=\operatorname{color}\left(M_{1}\right)$.

If $p=1$ then $H$ is a monochromatic triangle. The color of the triangle $H$ is color $\left(e_{1}\right)$. Since $E^{\prime}\left(e_{1}, V(H)\right) \neq \emptyset$, the monochromatic triangle $H$ contradicts Claim 5 .

If $p=2$ then we may assume that color $(a b)=\operatorname{color}\left(e_{1}\right)$ and $\operatorname{color}(a c)=\operatorname{color}(b c)=$ $\operatorname{color}\left(e_{2}\right)$. By Claim 4, we may assume that $x_{1} a \in E^{\prime}\left(e_{1}, V(H)\right)$ and $x_{2} c \in E^{\prime}\left(e_{2}, V(H)\right)$. (See Figure 6.) If there exists an edge $f \in E^{\prime}\left(y_{1}, e_{2}\right)$ then we have a rainbow $k$-matching

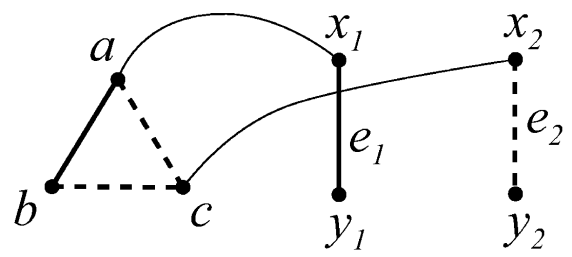

Figure 6: The case $p=2$.

$M-e_{1}-e_{2}+a x_{1}+b c+f$, which is a contradiction. Thus, $E^{\prime}\left(y_{1}, e_{2}\right)=\emptyset$. If there exists an edge $f \in E^{\prime}\left(y_{2}, e_{1}\right)$ then we have a rainbow $k$-matching $M-e_{1}-e_{2}+c x_{2}+a b+f$, which is a contradiction. Thus, $E^{\prime}\left(y_{2}, e_{1}\right)=\emptyset$. Hence, $E^{\prime}\left(e_{1}, e_{2}\right)=\left\{x_{1} x_{2}\right\}$, which implies that we could decrease one edge in the above counting argument. Therefore, we may assume that $\left|E^{\prime}\left(e_{2}, V(H)\right)\right|=2$. By Claim $4, E^{\prime}\left(e_{2}, V(H)\right)=\left\{c x_{2}, c y_{2}\right\}$. Then we have a rainbow $k$-matching $M-e_{1}-e_{2}+a b+c y_{2}+x_{1} x_{2}$, which is a contradiction.

If $p=3$ then we may assume that $\operatorname{color}(a b)=\operatorname{color}\left(e_{1}\right), \operatorname{color}(b c)=\operatorname{color}\left(e_{2}\right)$, and $\operatorname{color}(a c)=\operatorname{color}\left(e_{3}\right)$. (See Figure 7.) Without loss of generality, we may as-
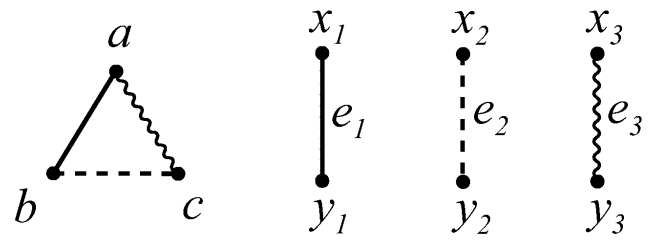

Figure 7: The case $p=3$.

sume that $\left|E^{\prime}\left(e_{1}, V(H)\right)\right|=2,\left|E^{\prime}\left(e_{2}, V(H)\right)\right|=2,\left|E^{\prime}\left(e_{3}, V(H)\right)\right| \geq 1$, otherwise we can decrease two edges in the counting argument. By Claim 4, $E^{\prime}\left(e_{1}, V(H)\right)=E^{\prime}\left(e_{1}, a b\right)$, 
$E^{\prime}\left(e_{2}, V(H)\right)=E^{\prime}\left(e_{2}, b c\right)$, and $E^{\prime}\left(e_{3}, V(H)\right)=E^{\prime}\left(e_{3}, a c\right)$. If the two edges in $E^{\prime}\left(e_{1}, V(H)\right)$ are independent, say, if $a x_{1}, b y_{1} \in E^{\prime}\left(e_{1}, V(H)\right)$, then we have a rainbow $k$-matching $M-e_{1}+a x_{1}+b y_{1}$, which is a contradiction. Suppose that $a x_{1}, a y_{1} \in E^{\prime}\left(e_{1}, V(H)\right)$. Without loss of generality, we may assume $x_{1} x_{2} \in E^{\prime}\left(e_{1}, e_{2}\right)$. Then we have a rainbow $k$-matching $M-e_{1}-e_{2}+x_{1} x_{2}+a y_{1}+b c$, which is a contradiction. Hence, we may assume that $a x_{1}, b x_{1} \in E^{\prime}\left(e_{1}, V(H)\right)$. Similarly for $e_{2}$, we may assume that $b x_{2}, c x_{2} \in$ $E^{\prime}\left(e_{2}, V(H)\right)$. If there exists an edge $f \in E^{\prime}\left(y_{1}, e_{2}\right)$ then we have a rainbow $k$-matching $M-e_{1}-e_{2}+a x_{1}+b c+f$, which is a contradiction. Thus, $E^{\prime}\left(y_{1}, e_{2}\right)=\emptyset$. If there exists an edge $f \in E^{\prime}\left(y_{2}, e_{1}\right)$ then we have a rainbow $k$-matching $M-e_{1}-e_{2}+c x_{2}+a b+f$, which is a contradiction. Thus, $E^{\prime}\left(y_{2}, e_{1}\right)=\emptyset$. Hence, $E^{\prime}\left(e_{1}, e_{2}\right)=\left\{x_{1} x_{2}\right\}$, which implies we can decrease one color in counting colors. Therefore, we may assume that $\left|E^{\prime}\left(e_{1}, e_{3}\right)\right|=\left|E^{\prime}\left(e_{2}, e_{3}\right)\right|=\mid E^{\prime}\left(e_{3}, V(H) \mid=2\right.$. Similarly as for $e_{1}, e_{2}$, we may assume that $a x_{3}, c x_{3} \in E^{\prime}\left(e_{3}, V(H)\right)$. If there exists an edge $f \in E^{\prime}\left(y_{2}, e_{3}\right)$ then we have a rainbow $k$-matching $M-e_{2}-e_{3}+b x_{2}+a c+f$, which is a contradiction. Thus, $E^{\prime}\left(y_{2}, e_{3}\right)=\emptyset$, which implies $x_{2} x_{3}, x_{2} y_{3} \in E^{\prime}\left(e_{2}, e_{3}\right)$. Then we have a rainbow $k$-matching $M-e_{2}-e_{3}+a x_{3}+b c+x_{2} y_{3}$, which is a contradiction.

Here, we classify the edges of $M_{2}$ as follows:

$$
\begin{aligned}
& M_{2,1}=\left\{e \in M_{2}|| E^{\prime}\left(e, V(H) \cup V_{1}\right) \mid \geq 2 p+1\right\}, \\
& M_{2,2}=M_{2}-M_{2,1} .
\end{aligned}
$$

Note that by Claim 3, for any edge $e \in M_{2,1}, E^{\prime}(e, V(H)) \neq \emptyset$. Let $V_{2,1}=\bigcup_{x y \in M_{2,1}}\{x, y\}$ and $V_{2,2}=\bigcup_{x y \in M_{2,2}}\{x, y\}$. (See Figure 8.)

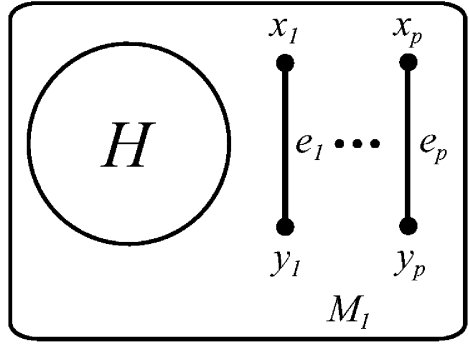

$V(H) \cup V_{l}$

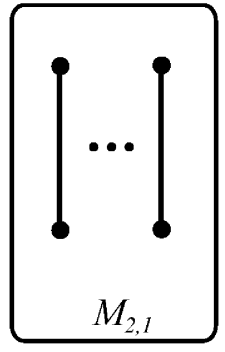

$V_{2,1}$

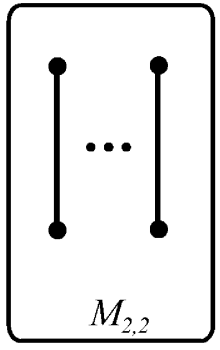

$V_{2,2}$

Figure 8: $H, M_{1}, M_{2,1}$, and $M_{2,2}$.

Claim 9. $\left|E^{\prime}\left(V(H) \cup V_{1}, V_{2,1}\right)\right| \leq(2 p+|V(H)|)\left|M_{2,1}\right|$.

Proof. By Claim 3, for any edge $e \in M_{2,1},\left|E^{\prime}\left(e, V_{1}\right)\right| \leq 2 p$. If there are two independent edges $f_{1}, f_{2} \in E^{\prime}(e, V(H))$ then we have a rainbow $k$-matching $M-e+f_{1}+f_{2}$. Thus, $\left|E^{\prime}(e, V(H))\right| \leq|V(H)|$ because $|V(H)| \neq 1$. Therefore $\left|E^{\prime}\left(V_{2,1}, V_{1} \cup V(H)\right)\right| \leq(2 p+$ $|V(H)|)\left|M_{2,1}\right|$. 
Claim 10. Let $e_{i}, e_{j}$ be two distinct edges in $M_{2}$. If both $E^{\prime}\left(e_{i}, V(H)\right)$ and $E^{\prime}\left(e_{j}, V(H)\right)$ are non-empty, and $\left|E^{\prime}\left(e_{i}, e_{j}\right)\right|=4$, then all edges in $E^{\prime}\left(e_{i}, V(H)\right)$ and $E^{\prime}\left(e_{j}, V(H)\right)$ are incident to exactly one vertex of $V(H)$.

Proof. Suppose that for two distinct vertices $a, b \in V(H), a x_{i}, b x_{j} \in E\left(G^{\prime}\right)$. Then we have a rainbow $k$-matching $M-e_{i}-e_{j}+a x_{i}+b x_{j}+y_{i} y_{j}$, which is a contradiction.

Claim 11. Let $e_{i}, e_{j}$ be two distinct edges in $M_{2}$. If $\left|E^{\prime}\left(e_{i}, V_{1}\right)\right| \geq 2 p-1$ and $E^{\prime}\left(e_{j}, V(H)\right)$ $\neq \emptyset$, then $\left|E^{\prime}\left(e_{i}, e_{j}\right)\right| \leq 3$.

Proof. Let $a \in V(H)$, and without loss of generality, we may assume $a x_{j} \in E^{\prime}\left(e_{j}, V(H)\right)$. Since $|V(H)| \geq 3, E(H-a) \neq \emptyset$. Let $b c \in E(H-a)$. Without loss of generality, we may assume that color $(b c)=\operatorname{color}\left(e_{1}\right)$. (See Figure 9.) By Claim 3 and our assumption that

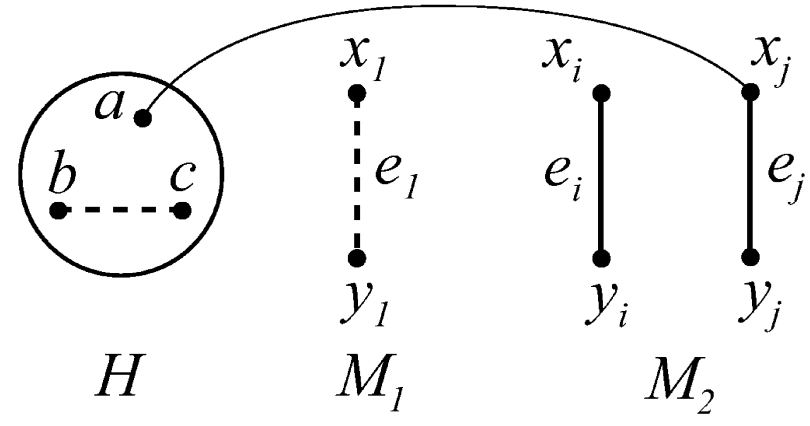

Figure 9: Proof of Claim 11.

$\left|E^{\prime}\left(e_{i}, V_{1}\right)\right| \geq 2 p-1, E^{\prime}\left(e_{i}, e\right) \neq \emptyset$ for any $e \in M_{1}$. Hence, without loss of generality, we may assume that $x_{i} x_{1} \in E^{\prime}\left(e_{i}, e_{1}\right)$. Suppose that $\left|E^{\prime}\left(e_{i}, e_{j}\right)\right|=4$. Then we have a rainbow $k$-matching $M-e_{i}-e_{j}-e_{1}+b c+a x_{j}+x_{i} x_{1}+y_{i} y_{j}$, which is a contradiction.

Claim 12. For any two distinct edges $e_{i}, e_{j} \in M_{2,1},\left|E^{\prime}\left(e_{i}, e_{j}\right)\right| \leq 3$.

Proof. By Claim 3 and the definition of $M_{2,1}, E^{\prime}\left(e_{i}, V(H)\right)$ and $E^{\prime}\left(e_{j}, V(H)\right)$ are not empty. Suppose that $\left|E^{\prime}\left(e_{i}, e_{j}\right)\right|=4$. By Claim 10, all edges in $E^{\prime}\left(e_{i}, V(H)\right)$ and $E^{\prime}\left(e_{j}, V(H)\right)$ are incident to exactly one vertex of $V(H)$. Thus, $\left|E^{\prime}\left(e_{i}, V(H)\right)\right| \leq 2$. Since $\left|E^{\prime}\left(e_{i}, V_{1} \cup V(H)\right)\right| \geq 2 p+1$ by the definition of $M_{2,1}$, we have $\left|E^{\prime}\left(e_{i}, V_{1}\right)\right| \geq 2 p-1$. Hence by Claim 11, $\left|E^{\prime}\left(e_{i}, e_{j}\right)\right| \leq 3$.

Claim 13. For any edge $e_{j} \in M_{2,2}$, there is at most one edge $e \in M_{2,1}$ such that $\left|E^{\prime}\left(e, e_{j}\right)\right|=4$.

Proof. Suppose that there are two distinct edges $e_{s}, e_{t} \in M_{2,1}$ such that $\left|E^{\prime}\left(e_{s}, e_{j}\right)\right|=4$ and $\left|E^{\prime}\left(e_{t}, e_{j}\right)\right|=4$. By Claim 3 and the definition of $M_{2,1}, E^{\prime}\left(e_{s}, V(H)\right)$ and $E^{\prime}\left(e_{t}, V(H)\right)$ are not empty. Let $x_{s} v \in E^{\prime}\left(e_{s}, V(H)\right)$ and $x_{t} v^{\prime} \in E^{\prime}\left(e_{t}, V(H)\right)$. If $v \neq v^{\prime}$ then we have a rainbow $k$-matching $M-e_{s}-e_{t}-e_{j}+v x_{s}+v^{\prime} x_{t}+y_{s} x_{j}+y_{t} y_{j}$, which is a contradiction. Thus, $v=v^{\prime}$ and $\left|E^{\prime}\left(e_{s}, V(H)\right)\right| \leq 2$. Then by the definition of $M_{2,1}$, we have $\left|E^{\prime}\left(e_{s}, V_{1}\right)\right| \geq 2 p-1$. 
Hence, for any edge $e \in M_{1}, E^{\prime}\left(e, e_{s}\right) \neq \emptyset$. Let $a b \in E(H-v)$. There is an edge $e \in M_{1}$, say $e_{1}$, such that color $\left(e_{1}\right)=\operatorname{color}(a b)$. (See Figure 10.)

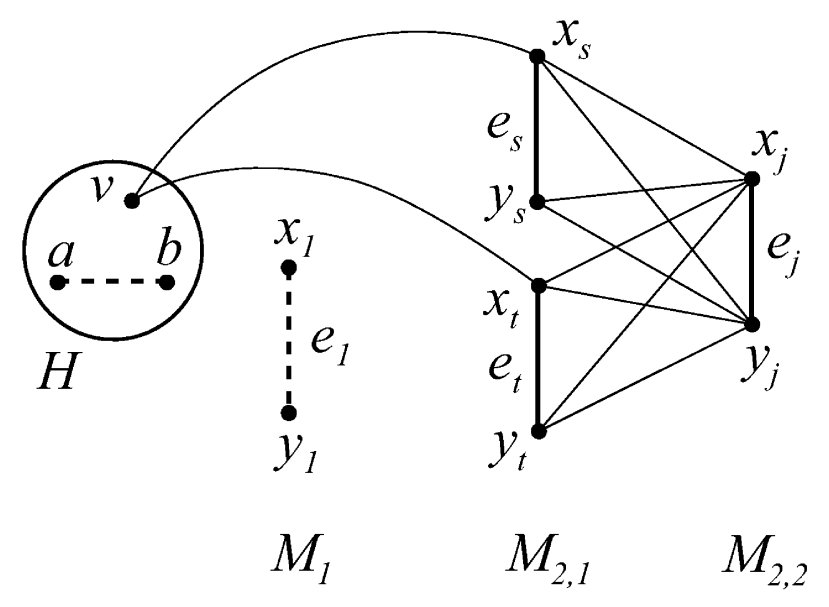

Figure 10: Proof of Claim 13.

Recall $E^{\prime}\left(e_{1}, e_{s}\right) \neq \emptyset$. Utilizing this fact, we can easily find a rainbow $k$-matching. To see this, say, assume that $x_{1} x_{s} \in E^{\prime}\left(e_{1}, e_{s}\right)$. Then we have a rainbow $k$-matching $M-e_{s}-e_{t}-e_{j}-e_{1}+a b+v x_{t}+x_{1} x_{s}+y_{s} x_{j}+y_{t} y_{j}$, which is a contradiction. We can similarly get a contradiction in other cases. Thus the claim holds.

Claim 14. $\left|E^{\prime}\left(V_{2,2}, V(H) \cup V_{1} \cup V_{2,1}\right)\right| \leq\left(2 p+3\left|M_{2,1}\right|\right)\left|M_{2,2}\right|$.

Proof. Let $e_{j} \in M_{2,2}$. By the definition of $\left.M_{2,2}, \mid E^{\prime}\left(e_{j}, V(H) \cup V_{1}\right)\right) \mid \leq 2 p$. If for any edge $e_{i} \in M_{2,1},\left|E^{\prime}\left(e_{i}, e_{j}\right)\right| \leq 3$ holds, then we have $\left|E^{\prime}\left(e_{j}, V(H) \cup V_{1} \cup V_{2,1}\right)\right| \leq 2 p+3\left|M_{2,1}\right|$.

By Claim 13, there is at most one edge $e_{i} \in M_{2,1}$ such that $\left|E^{\prime}\left(e_{i}, e_{j}\right)\right|=4$. Suppose that there exists exactly one edge $e_{i} \in M_{2,1}$ such that $\left|E^{\prime}\left(e_{i}, e_{j}\right)\right|=4$. By Claim 3 and the definition of $M_{2,1}, E^{\prime}\left(e_{i}, V(H)\right) \neq \emptyset$. Let $x_{i} v \in E^{\prime}\left(e_{i}, V(H)\right)$. Suppose $E^{\prime}\left(e_{j}, V(H)\right) \neq \emptyset$. Then by Claim 10, all edges in $E^{\prime}\left(e_{i}, V(H)\right)$ and $E^{\prime}\left(e_{j}, V(H)\right)$ are incident to $v$. Thus, $\left|E^{\prime}\left(e_{i}, V(H)\right)\right| \leq 2$. By the definition of $M_{2,1},\left|E^{\prime}\left(e_{i}, V(H) \cup V_{1}\right)\right| \geq 2 p+1$. Hence $\left|E^{\prime}\left(e_{i}, V_{1}\right)\right| \geq 2 p-1$. Therefore, by Claim 11, $\left|E^{\prime}\left(e_{i}, e_{j}\right)\right| \leq 3$, which is a contradiction. Hence we may assume that $E^{\prime}\left(e_{j}, V(H)\right)=\emptyset$. Then, by Claim $11,\left|E^{\prime}\left(e_{j}, V_{1}\right)\right| \leq 2 p-2$. Thus, $\left|E^{\prime}\left(e_{j}, V(H) \cup V_{1} \cup V_{2,1}\right)\right| \leq 2 p-2+3\left(\left|M_{2,1}\right|-1\right)+4=2 p+3\left|M_{2,1}\right|-1$.

Consequently, for any $e_{j} \in M_{2,2}$, we have $\left|E^{\prime}\left(e_{j}, V(H) \cup V_{1} \cup V_{2,1}\right)\right| \leq 2 p+3\left|M_{2,1}\right|$. Hence, the Claim holds.

Recall that $r=|\operatorname{color}(G)|=\left|\operatorname{color}\left(G^{\prime}\right)\right|=\left|E\left(G^{\prime}\right)\right|$. We prove that

$$
\left|E\left(G^{\prime}\right)\right|<\max \left\{\left(\begin{array}{c}
2 k-3 \\
2
\end{array}\right)+2,\left(\begin{array}{c}
k-2 \\
2
\end{array}\right)+(k-2)(n-k+2)+2\right\}
$$

by the above Claims. 
Now, we have

$$
\begin{aligned}
\left|E\left(G^{\prime}\right)\right|= & \left.\left|E\left(G^{\prime}\left[V(H) \cup V_{1}\right]\right)\right|+\left|E^{\prime}\left(V(H) \cup V_{1}, V_{2,1}\right)\right|+\mid E\left(G^{\prime}\left[V_{2,1}\right]\right)\right) \mid \\
& \left.+\left|E^{\prime}\left(V(H) \cup V_{1} \cup V_{2,1}, V_{2,2}\right)\right|+\mid E\left(G^{\prime}\left[V_{2,2}\right]\right)\right) \mid .
\end{aligned}
$$

By Claim 8, $\left|E\left(G^{\prime}\left[V(H) \cup V_{1}\right]\right)\right| \leq 2\left(\begin{array}{c}p \\ 2\end{array}\right)+3 p-2$. By Claim 9, $\left|E^{\prime}\left(V(H) \cup V_{1}, V_{2,1}\right)\right| \leq$ $(2 p+|V(H)|)\left|M_{2,1}\right|$. By Claim 12, $\left.\mid E\left(G^{\prime}\left[V_{2,1}\right]\right)\right)\left|\leq 3\left(\begin{array}{c}\left|M_{2,1}\right| \\ 2\end{array}\right)+\right| M_{2,1} \mid$. By Claim 14, $\left|E^{\prime}\left(V(H) \cup V_{1} \cup V_{2,1}, V_{2,2}\right)\right| \leq\left(2 p+3\left|M_{2,1}\right|\right)\left|M_{2,2}\right|$. Also, the number of edges of $G^{\prime}\left[V_{2,2}\right]$ is upper bounded by the number of edges of the complete graph on $V_{2,2}$. Since $\left|V_{2,2}\right|=$ $2\left|M_{2,2}\right|$, it follows that $\left|E\left(G^{\prime}\left[V_{2,2}\right]\right)\right| \leq\left(\begin{array}{c}2\left|M_{2,2}\right| \\ 2\end{array}\right)$. Therefore, we have

$$
\begin{aligned}
\left|E\left(G^{\prime}\right)\right| \leq & 2\left(\begin{array}{l}
p \\
2
\end{array}\right)+3 p-2+(2 p+|V(H)|)\left|M_{2,1}\right|+3\left(\begin{array}{c}
\left|M_{2,1}\right| \\
2
\end{array}\right)+\left|M_{2,1}\right| \\
& +\left(2 p+3\left|M_{2,1}\right|\right)\left|M_{2,2}\right|+\left(\begin{array}{c}
2\left|M_{2,2}\right| \\
2
\end{array}\right) .
\end{aligned}
$$

Let $q=\left|M_{2,1}\right|$ and $h=|V(H)|$, then $\left|M_{2,2}\right|=|M|-\left|M_{1}\right|-\left|M_{2,1}\right|=k-1-p-q$. Hence,

$$
\begin{aligned}
\left|E\left(G^{\prime}\right)\right| \leq & 2\left(\begin{array}{l}
p \\
2
\end{array}\right)+3 p-2+(2 p+h) q+3\left(\begin{array}{l}
q \\
2
\end{array}\right)+q \\
& +(2 p+3 q)(k-1-p-q)+\left(\begin{array}{c}
2(k-1-p-q) \\
2
\end{array}\right) \\
= & \frac{1}{2} q^{2}+\left(h-k+p+\frac{3}{2}\right) q+p^{2}+(5-2 k) p+2 k^{2}-5 k+1 \\
= & \frac{1}{2}\left(q+h-k+p+\frac{3}{2}\right)^{2}-\frac{1}{2}\left(h-k+p+\frac{3}{2}\right)^{2}+p^{2}+(5-2 k) p+2 k^{2}-5 k+1 \\
= & \frac{1}{2}\left(q+h-k+p+\frac{3}{2}\right)^{2}+\frac{1}{2} p^{2}+\left(\frac{7}{2}-h-k\right) p \\
& -\frac{1}{2} h^{2}+\left(k-\frac{3}{2}\right) h+\frac{3}{2} k^{2}-\frac{7}{2} k-\frac{1}{8} .
\end{aligned}
$$

Let $F(q, p)$ be a function with two parameter $q$ and $p$ as follows:

$$
\begin{aligned}
F(q, p)= & \frac{1}{2}\left(q+h-k+p+\frac{3}{2}\right)^{2}+\frac{1}{2} p^{2}+\left(\frac{7}{2}-h-k\right) p \\
& -\frac{1}{2} h^{2}+\left(k-\frac{3}{2}\right) h+\frac{3}{2} k^{2}-\frac{7}{2} k-\frac{1}{8} .
\end{aligned}
$$

For this function $F(q, p)$, we do quadratic optimization by fixing the parameter $p$, that is, we assume $F(q)=F(q, p)$ is a quadratic function with the parameter $q$. Note that $0 \leq q=\left|M_{2,1}\right| \leq\left|M_{2}\right|=|M|-\left|M_{1}\right|=k-1-p$. Then, this function is maximum when $q=0$ or $q=k-1-p$. From (2), the corresponding value to the axis of symmetry of $F(q)$ is $q=-h+k-p-\frac{3}{2}$.

If the middle value of the range $0 \leq q \leq k-1-p$ is less than the corresponding value to the axis of symmetry, that is,

$$
\frac{0+k-1-p}{2} \leq-h+k-p-\frac{3}{2}
$$


then $F(q)$ is maximum when $q=0$. Since $h=|V(H)| \geq 3$, this together with (3) shows $p \leq k-2-2 h \leq k-8$. Hence, if $1 \leq p \leq k-8$ then $F(q)$ is maximum when $q=0$. From (1),

$$
F(0, p)=p^{2}+(5-2 k) p+2 k^{2}-5 k+1=\left(p+\frac{5-2 k}{2}\right)^{2}+k^{2}-\frac{21}{4} .
$$

This is a function with the parameter $p$ whose axis of symmetry is $p=(2 k-5) / 2$. Since, the middle value of the range $1 \leq p \leq k-8$ is less than the corresponding value to the axis of symmetry, $F(0, p)$ is maximum when $p=1$.

$$
F(0,1)=2 k^{2}-7 k+7<2 k^{2}-7 k+8=\left(\begin{array}{c}
2 k-3 \\
2
\end{array}\right)+2 .
$$

Hence $\left|E\left(G^{\prime}\right)\right|<\max \left\{\left(\begin{array}{c}2 k-3 \\ 2\end{array}\right)+2,\left(\begin{array}{c}k-2 \\ 2\end{array}\right)+(k-2)(n-k+2)+2\right\}$. Therefore, we may assume that $F(q, p)$ is maximum when $q=k-1-p$.

$$
\begin{aligned}
F(k-1-p, p) & =\frac{1}{2} p^{2}+\left(\frac{7}{2}-h-k\right) p+(k-1) h+\frac{3}{2} k^{2}-\frac{7}{2} k \\
& =\frac{1}{2}\left(p+\frac{7}{2}-h-k\right)^{2}+\frac{1}{2}\left(\frac{7}{2}-h-k\right)^{2}+(k-1) h+\frac{3}{2} k^{2}-\frac{7}{2} k .
\end{aligned}
$$

This is a function with the parameter $p$ whose axis of symmetry is $p=k+h-7 / 2$. Since the middle value of the range $1 \leq p \leq k-1$ is less than the corresponding value to the axis of symmetry, $F(k-1-p, p)$ is maximum when $p=1$.

$$
F(k-1-p, 1)=\frac{3}{2} k^{2}-\frac{9}{2} k+4+(k-2) h .
$$

Since $n=|V(H)|+2|M|=h+2(k-1)$, that is $h=n-2 k+2$, we have

$$
\begin{aligned}
F(k-1-p, 1) & =\frac{3}{2} k^{2}-\frac{9}{2} k+4+(k-2)(n-2 k+2) \\
& =\frac{1}{2} k^{2}-\frac{5}{2} k+4+(k-2)(n-k+2) \\
& <\frac{1}{2} k^{2}-\frac{5}{2} k+5+(k-2)(n-k+2) \\
& =\left(\begin{array}{c}
k-2 \\
2
\end{array}\right)+(k-2)(n-k+2)+2 .
\end{aligned}
$$

Hence $\left|E\left(G^{\prime}\right)\right|<\max \left\{\left(\begin{array}{c}2 k-3 \\ 2\end{array}\right)+2,\left(\begin{array}{c}k-2 \\ 2\end{array}\right)+(k-2)(n-k+2)+2\right\}$.

\section{Acknowledgments}

We would like to thank Prof. Akira Saito of Nihon University for many helpful discussions, and the referee for many valuable comments for improving the presentation in the previous version of this paper. Also, this work is partially supported by the JSPS Research Fellowships for Young Scientists (to S.F). 


\section{References}

[1] Erdős, P.; Gallai, T.: On maximal paths and circuits of graphs, Acta Math. Acad. Sci. Hungar 10 (1959), 337-356.

[2] Erdős, P.; Simonovits, M.; Sós, V.T.: Anti-Ramsey theorems, Infinite and finite sets (Colloq., Keszthely, 1973; dedicated to P.Erdös on his 60th birthday), Vol. II, (1975), Colloq. Math. Soc. János Bolyai, Vol. 10, North-Holland, Amsterdam, 633-643.

[3] Eroh, Linda: Rainbow Ramsey numbers of stars and matchings, Bull. Inst. Combin. Appl. 40 (2004), 91-99.

[4] Eroh, Linda: Constrained Ramsey numbers of matchings, J. Combin. Math. Combin. Comput. 51 (2004), 175-190.

[5] Montellano-Ballesteros, J.J.; Neumann-Lara, V.: An anti-Ramsey theorem, Combinatorica 22 (2002), no.3, 445-449.

[6] Schiermeyer, Ingo: Rainbow numbers for matchings and complete graphs, Discrete Math. 286 (2004), no.1-2, 157-162. 